\title{
Interleaving and Termination in Unpunctured Symmetric Turbo Codes
}

\author{
Johann A. Briffa and Victor Buttigieg \\ Department of Communications and Computer Engineering \\ University of Malta \\ Msida MSD 06, MALTA \\ E-mail: jabrif@eng.um.edu.mt
}




\begin{abstract}
The Turbo code interleaver design problem is considered for large block sizes, where the effect of trellis termination is less marked. The performance of various interleavers with a similar block size are compared, including an implementation of the uniform interleaver. An optimised interleaver design technique based on simulated annealing is proposed - the results obtained show that the error performance may be significantly improved without increasing the delay. Finally, interleaver design for small Turbo codes is considered. In this case it is shown that while correct termination improves the performance for an average interleaver, its effect on Turbo codes with optimised interleavers is negligible.

Using our simulated annealing design technique it is easier to include restrictions which make the interleaver correctly-terminating or odd-even. While the $S$-random algorithm serves well for specifying interleaver spread, we believe that our algorithm is better suited for more sophisticated design criteria.
\end{abstract}

\title{
Keywords
}

Turbo codes, interleaver design, trellis termination, simulated annealing.

\section{INTRODUCTION}

\section{A. Classical Use of Interleavers}

Interleavers have been used in communication systems for a long time; the classical use is to randomise the location of errors, enabling the use of random error-correcting codes on channels with burst error patterns. Typically, bursty channels would include fading channels often found in wireless transmission. Another use of interleaving is in concatenated coding, where the output of the inner-stage decoder exhibits burst error patterns (as happens with a Viterbi decoder). Hence, the most important parameter of the interleaver in this case is its ability to spread error bursts such that they appear as isolated errors to the outer-stage decoder. Naturally, the optimum interleaver would achieve this with the minimum memory [1]. It should be clear that the required parameters of the interleaver in this case depend uniquely on the inner and outer codes and decoders used.

\section{B. Interleavers in Turbo Codes}

Turbo coding, however, also introduced a further dimension to what is required from the interleaver - this involves the effects of the iterative algorithm and the passing of extrinsic information between successive decoder stages. In this context, the interleaver has often 
been explained as reducing the correlation between the parity bits corresponding to the original and interleaved data frames. However, for reasons which will be explained later, this terminology isn't quite accurate.

In the original paper introducing Turbo codes, Berrou et al. [2] already showed an exceptional understanding of some of the most important parameters making a good interleaver. In particular:

- Increasing the block size (and hence the size of the interleaver) results in improved performance.

- The interleaver should randomise the input sequence in order to avoid particular lowweight patterns mapping onto themselves, reducing the effective free distance of the resulting Turbo Code.

\section{Paper Overview}

The rest of this paper is divided as follows: the next section gives a brief literature review of interleaver design for Turbo codes. This is followed by a description of our proposed interleaver design technique based on simulated annealing. Next, we compare our design technique with others in the literature. This is done first for large block sizes, where the effect of termination can be ignored. Then we consider the effect of termination in small Turbo codes with optimised and standard interleavers. Finally, we give our conclusions.

\section{Brief Literature Review}

While the effect of the interleaver on Turbo code performance was recognised early [3], the sheer size of the problem of choosing an optimum interleaver meant that a comprehensize solution remained elusive. It was already shown, however, that randomly chosen interleavers perform better than structured interleavers. The actual importance of randomisation was shown by Divsalar and Pollara [4] and Perez et al. [5], by considering the effect of the interleaver on critical low weight input patterns. Divsalar and Pollara [4] also proposed the $S$-random interleaver, which is a randomly created interleaver with some restrictions, designed to break low-weight input patterns. Other semi-random interleaver designs include the original one used by Berrou and Glavieux [6].

Other early attempts at interleaver design include those by Jung and Naßhan [7]. In 
this paper, interleavers are chosen from a large set of random interleavers based on the low-weight-input distance properties of the resulting Turbo code. It was stated that a regular block interleaver performs better than the chosen random interleaver for the given block size. This contrasts with the opinion given elsewhere in the literature ${ }^{1}$ that regular interleavers should be avoided because of their poor distance properties.

Recently, a mathematical model of interleavers has been proposed by Andrews et al. [8], who also used this to guide the generation of new interleavers for Turbo codes by modifying the $S$-random algorithm [9]. It is interesting to note that most papers on interleaver design are based on the $S$-random algorithm, which was designed to counter the effects of weight-2 input sequences only.

A more recent interleaver design technique has been presented by Hokfelt et al.[10]. Their design technique is based on the statistical properties of the extrinsic information passed between decoders rather than on the Turbo code's distance properties. Another recent proposal by Yuan et al.[11], [12] is a modification of the $S$-random interleaver design which also takes into account the first few terms of the distance spectrum of the component codes.

\section{Optimised Interleaver Design}

\section{A. Interleaver Input-Output Separation Spectrum}

An interleaver is said to have a spread $S$ if any bit-pairs that are $S$ or fewer places apart at the input will be at least $S$ places apart at the output of the interleaver. In order to visualise this spread and how it is achieved in any particular interleaver, we plot the Input-Output Separation Spectrum (IOSS). This graph is a two-dimensional histogram, with the axes being the separation for all possible bit-pairs at the input and output of the interleaver; the darker the region the more instances (multiplicity) with the given parameters. For comparison, we plot the IOSS (limiting ourselves to a maximum separation of 100) for a Square and Berrou-Glavieux interleaver of equal size in Figs. 2 and 3 respectively. These interleavers are used in Section IV, and are detailed in Table I.

Note how the Square interleaver concentrates bit-pairs in very small regions, while the

${ }^{1}$ See, for example, [4]. 
Berrou-Glavieux interleaver (through the pseudo-random perturbations) spreads out the regions. For this reason, the peak multiplicity for the Square interleaver is almost four times that of the Berrou-Glavieux interleaver. Note that the concentration of points for the Square interleaver is indicative of its lack of randomness, which is the major cause for its poor performance.

\section{B. S-random Interleaver}

The $S$-random interleaver design is a randomly chosen interleaver with a restriction on the spread $S$. The searching time for this algorithm increases with $S$ and the algorithm is not guaranteed to finish successfully. Divsalar and Pollara [4] have observed, however,

that for an interleaver of size $\tau$, choosing $S<\sqrt{\frac{\tau}{2}}$ usually produces a result in a reasonable time.

The main problems with this technique are that it is not guaranteed to produce the required interleaver and that it only aims at achieving a spread $S$. Seen in IOSS space, this means that a square of side $S$ starting at the origin must be kept empty. The distribution of points outside this square is not considered. Usually, with a large interleaver that is randomly chosen, the distribution outside the spread boundary is approximately flat. This can be seen in Fig. 4, which shows the IOSS for an $S$-random interleaver of size $\tau=1024$. Compared with interleavers having higher regularity, such as the BerrouGlavieux interleaver of Fig. 3, the flattening of the IOSS reduces the peak multiplicity. For example, the peak multiplicity of the Berrou-Glavieux interleaver is about eight times that of the $S$-random interleaver.

\section{Simulated Annealing Interleaver}

The Simulated Annealing (SA) algorithm for global minimisation is now a well-proven and accepted technique, and has been used successfully in various applications for minimisation of a combinatorial (rather than a continuous) function [13]. The method is analogous to the way that slowly cooled crystals achieve a state of minimum energy in thermodynamics.

In order to simultaneously optimise the various requirements for our interleaver we define an energy function based on these requirements, such that a lower energy corresponds 
to a better-performing interleaver. We then use the simulated annealing algorithm to minimise the energy function. Although this is not guaranteed to converge to a globally optimal solution ${ }^{2}$, in our experience simulated annealing will converge to a good suboptimal solution in various optimisation problems with large dimensionality (as in this case). There is also the advantage that the resulting interleaver appears similar to a random one.

\section{C.1 Simulated Annealing Algorithm}

In the physical counterpart, a solid is first heated up to a temperature at which all its particles randomly arrange themselves in the liquid state. Next the temperature is slowly lowered until the particles arrange themselves in a low-energy ground state. It is essential that the initial temperature is sufficiently high and that the temperature is reduced sufficiently slowly, to ensure that defects are not frozen into the solid. At each temperature $T$, the solid is allowed to reach thermal equilibrium, such that the energy distribution is given by the Boltzmann distribution:

$$
\operatorname{Pr}[\text { Energy }=E]=\frac{1}{Z(T)} \cdot \exp \left(-\frac{E}{k_{B} T}\right)
$$

where $Z(T)$ is a normalisation factor and $k_{B}$ is the Boltzmann constant. This process is simulated by the Metropolis Algorithm [13], where at any particular temperature $T$ a perturbation resulting in a decrease in energy is always accepted, while an increase is only accepted with a probability $\exp \left(\frac{\Delta E}{T}\right)$, where $\Delta E$ is the increase in energy. Temperature in the simulation is a virtual value which also absorbs the effect of the Boltzmann constant in the physical counterpart. A large number of random perturbations are allowed until equilibrium is reached; for successive iterations the temperature is decreased exponentially, until the final low energy state is reached. It can be shown that in the limit, as $T \rightarrow 0$, the global minimum is reached. The basic algorithm is outlined in Fig. 1. We will now consider the parameters that can be varied in the algorithm:

- The energy function - since this is the function that is minimised by the algorithm, it needs to reflect the performance of the interleaver. We start by adapting the 'high

${ }^{2}$ The algorithm does converge asymptotically to the global optimum - however, implementation constraints will necessarily inhibit this. 
spreading factor' criterion used by Divsalar and Pollara in their $S$-random interleaver. While we aim for a high spreading factor (at least $5 \nu$, where $\nu$ is the component code's memory order ${ }^{3}$ ), we also want the worst-case spreads to have low multiplicity. We do this by minimising the number of points close to the origin in the interleaver's IOSS. With this in mind, we choose an energy function which sums over all points in the IOSS, with increased weight on points close to the origin. This attempts to 'push' bit-pair points away from the origin, thereby increasing the spread of the interleaver. One particular energy function that can be used is:

$$
E=\sum_{i, j} \frac{5 . \nu}{\sqrt{(i-j)^{2}+[\lambda(i)-\lambda(j)]^{2}}}
$$

where $i, j \in[0, \tau-1], i>j, \tau$ is the interleaver's size, $\nu$ is the encoder memory, and $\lambda()$ is the interleaving function. Note that the denominator $\sqrt{(i-j)^{2}+[\lambda(i)-\lambda(j)]^{2}}$ is the radial distance from the origin of the point described by the bit pair $i, j$.

- The perturbation scheme - it is generally preferred to have a perturbation scheme chosen such that the variation of energy with every perturbation be as smooth as possible. Also, the perturbation scheme must be chosen so as not to exclude any part of the search space. We meet these conditions in our case by restricting perturbations to a swap of two random interleaver entries. It is not difficult to constrain the interleaver in order to make it correctly-terminating, by choosing an appropriate perturbation scheme (and initial assignment) [14].

- The initial code assignment $X_{0}$ - this should not make any difference in the result if the other parameters are chosen correctly. This is because at the initial (sufficiently high) temperature, the system's energy will rise considerably as most perturbations will be accepted. Thus, a random interleaver should be as good as any other. This has been verified experimentally.

- The initial and final temperatures - to allow the system to migrate towards a highenergy state in the initial phase, the starting temperature should be chosen to be much greater than the average change in energy due to a single perturbation. This ensures that perturbations which increase the energy have a high probability of acceptance at initial

${ }^{3}$ The limit of $5 \nu$ is reached after analysing the confidence at the output of a MAP decoder for an elementary half-rate code when the input had a single hard error. 
temperatures. Note that the choice of a good perturbation scheme is necessary such that $\Delta E$ has a sufficiently restricted variance.

The algorithm stops when the state configuration becomes stable. This can be implemented by stopping the algorithm after, say, five reductions in temperature occur without any new state being accepted. In the general case, this is enough, but a minimum temperature condition is usually also applied. This minimum temperature should be chosen such that it is sufficiently lower than the temperatures at which the configuration becomes stable in the normal case, to ensure that we do not stop prematurely.

- The annealing schedule - since the probability of acceptance of an uphill move decreases exponentially with temperature, the annealing schedule is generally a geometric decrease of temperature $T_{i+1}=\alpha T_{i}$, where $\alpha$ lies in the range $0.90 \rightarrow 0.99$. Values of $\alpha$ closer to 1.0 make the temperature decrease more slowly, and generally results in better annealing, with a penalty to be paid as an increased computation time.

- The number of iterations allowed at every temperature - this should be large enough to allow the configuration to reach an energy state typical of that temperature. In practice, the number of iterations should be such that a significant proportion of the possible perturbations are tried. The only adverse effect of choosing too high a value is a proportional increase in computation time. It may be noted that this value is particularly important at low temperatures, where very few state changes are in fact accepted. At high temperatures, most proposed changes will be accepted, so the neccessary number of iterations is lower at high temperatures. This is achieved by adding another limit to the number of iterations - the maximum number of accepted state changes, which should be lower then the maximum number of iterations, typically by an order of magnitude. Thus, at high temperatures, the number of iterations performed is limited by the number of accepted state changes, while at lower temperatures, it is limited by the maximum number of iterations.

\section{C.2 Comparison of Simulated Annealing with $S$-random Interleavers}

In contrast with the $S$-random technique, our algorithm does not guarantee a particular spread. On the other hand, it pushes points away from the origin even beyond the spread boundary. The IOSS for an interleaver designed using Simulated Annealing is shown in 
Fig. 5. For comparison, see also the IOSS for an $S$-random interleaver in Fig. 4. The IOSS peak multiplicity of the Simulated Annealing interleaver is similar to that of the $S$-random interleaver. Note how the peak multiplicity for the Simulated Annealing interleaver has a radial shape. This is expected since the algorithm attempts to 'push' bit-pairs away from the origin.

\section{INTERLEAVER Design for LARge Frames}

In this section we consider the interleaver design problem for large block sizes, where the effect of trellis termination is less marked. This is done by comparing the performance of various interleavers with a similar block size. Finally, the performance of an optimised interleaver design technique based on simulated annealing is considered.

\section{A. Performance Reference}

We restrict ourselves to unpunctured rate- $\frac{1}{3}$ symmetric Turbo codes with encoder memory $\nu=2$ and generator $(1,5 / 7)$. In order to avoid the effects of trellis termination, we also choose a relatively large block size $\tau=1024$. The effect of interleaver choice for Turbo codes with this component code and block size are indicative of what can be expected with other good component codes and larger block sizes; this has been verified experimentally. The design problem of choosing the best component codes has been tackled by the JPL team [15].

As a reference for performance, we implement a uniform interleaver by using a different random (equiprobable) interleaver for every block simulated. To confirm the validity of the statement that trellis termination does not have a significant effect at the chosen block size, we simulate the uniform interleaver with and without termination. To terminate the uniform interleaver we use the scheme proposed by the JPL team [3]. The Bit Error Rate (BER) results for these two Turbo codes (E and F in Table I) are shown in Fig. 6; all simulations were performed using 10 iterations, with a target tolerance of $\pm 10 \%$ at a confidence of $95 \%$. As expected, trellis termination does not significantly affect the performance of Turbo codes with encoder memory $\nu=2$ at this block size. The Frame Error Rate (FER) performance is comparable to the BER performance. 


\section{B. Regular Interleavers}

It has mostly been argued that interleavers with a high regularity perform poorly in Turbo codes. This is confirmed when we simulate Turbo codes with Square, Rectangular, and Helical interleavers (codes A, B, and C in Table I), and compare with the performance of a uniform interleaver (code E), as shown in Fig. 6. Note that the Rectangular and Helical interleavers satisfy all the restrictions detailed in [1] and [16] respectively:

Rectangular: for the interleaver with $R=21$ rows and $C=49$ columns,

- $R+1$ and $C$ are relatively prime and $R+1<C$. These are conditions from [1] which give the interleaver a spread of $(R, C)$. Note that a Square interleaver cannot obey the latter condition.

- The interleaver spread is greater than $5 \nu$.

- Additionally, $R$ and $C$ are both odd, so that the interleaver is odd-even. This allows the same interleaver to be fairly compared with other such interleavers in a study of punctured Turbo codes.

Helical: for the interleaver with $R=29$ rows and $C=36$ columns,

- $R$ and $C$ are relatively prime.

- $C$ is a multiple of $\nu+1$ and the Recursive Systematic Convolutional (RSC) code's feedback polynomial is full, making the interleaver simile. This allows the same tail to be used with both the interleaved and non-interleaved sequences. In our implementation, we use the Tail Not Interleaved scheme proposed in [17], which was shown to give better results.

- Additionally, $C$ is even, so that the interleaver is odd-even.

It is interesting to note that the BER performance improvement of the Helical interleaver over the Square and Rectangular interleavers is minimal. Its FER performance, however, is somewhat better, though still far from the uniform interleaver.

\section{Randomised Interleavers}

The interleaver originally used by Berrou et al., described in [6], is essentially a Square interleaver with some pseudo-random perturbations. However, its performance is significantly better than a regular Square interleaver with the same dimensions. This is seen by 
comparing the Berrou-Glavieux interleaver (code D) and the Square interleaver (code A) in Fig. 6.

\section{Optimised Interleaver Design}

The performance of a Turbo code using the Simulated Annealing interleaver (code G) is shown in Fig. 7. For comparison, codes using the uniform interleaver (code E), the Berrou-Glavieux interleaver (code D), and an $S$-random interleaver (code H) are also shown. As can be seen from the graphs, optimising the interleaver improves performance when compared with partly-randomised designs. Code G can achieve a BER of $10^{-5}$ at

$\frac{E_{b}}{N_{0}}=1.35 \mathrm{~dB}$. It can also be seen that the performance of the Simulated Annealing interleaver is very similar to that of the $S$-random interleaver. This is expected because the interleaver for code $G$ was designed with much the same specifications as the basic $S$-random interleaver; the advantage of the Simulated Annealing technique, however, is that it is more flexible for the inclusion of additional design criteria, such as making an interleaver self-terminating, odd-even, and also facilitates the combined design of multiple interleavers for Turbo codes with more than two component codes.

Other energy functions based on the radial distance from the origin of IOSS points have also been tried, with very similar results. It seems that the combinatorial restrictions imposed on the interleaver structure do not allow significantly better interleavers (in the sense of increased distance of IOSS points from the origin) to be constructed. If there is anything further to be gained in interleaver design for Turbo codes, other factors need to be considered (such as puncturing, or higher-order distance statistics).

\section{Interleaver Design for Small Frames}

In this section we consider the interleaver design problem for small block sizes. The effect of various trellis termination schemes is analysed by comparing the performance of the uniform interleaver with and without termination. We also analyse the performance of different termination schemes when used with an optimised interleaver. 


\section{A. Performance Reference}

As in the previous section we restrict ourselves to unpunctured rate- $\frac{1}{3}$ symmetric Turbo codes with encoder memory $\nu=2$ and generator $(1,5 / 7)$. In contrast, though, we choose a very small block size $\tau=64$ to analyse the effect of termination where it should be most pronounced. The effect of interleaver choice for Turbo codes with this component code and block size are indicative of what can be expected with other good component codes and similarly small block sizes; this has been verified experimentally.

We simulate the uniform interleaver with and without termination. To terminate the uniform interleaver we use the scheme proposed by the JPL team. The Bit Error Rate (BER) and Frame Error Rate (FER) results for these two Turbo codes (A and B in Table II) are shown in Fig. 8; all simulations were performed using 10 iterations, with a target tolerance of $\pm 10 \%$ at a confidence of $95 \%$. While at low SNR the performance of the two codes is almost identical, at high SNR trellis termination improves the performance of Turbo codes with encoder memory $\nu=2$ at this block size. In particular, the FER performance is markedly better and the BER performance is marginally improved.

\section{B. Deterministic Interleavers}

The performance of Turbo codes with Square, Berrou-Glavieux, and Helical interleaver types (codes C, D, and E in Table II) are shown in Fig. 9 - FER performance is comparable to BER performance. For comparison, the performance of the terminated uniform interleaver (code B) is also shown in the graph. The Helical interleaver satisfies all necessary restrictions, as described in Section IV-B.

As for Turbo codes with large interleavers, the Square interleaver performs worst and the Berrou-Glavieux interleaver performs better than the uniform interleaver. In this case, however, the Helical interleaver performs better than the uniform interleaver both in terms of BER and FER.

\section{Optimised Interleaver Design}

The performance of Turbo codes with interleavers designed using Simulated Annealing (codes F, G, and H) are shown in Fig. 10 - FER performance is comparable to BER performance. For comparison, codes using the terminated uniform interleaver (code B) 
and the Berrou-Glavieux interleaver (code D) are also shown. As can be seen from the graph, optimising the interleaver improves performance. However, with an optimised interleaver design, the effect of termination becomes negligible, both when the JPL termination scheme is used with a non-terminating interleaver (code G) and also when a self-terminating interleaver is designed (code H). Consider both cases separately:

- When the JPL termination scheme is used in code $G$, the same interleaver is used as

the one for code F. The only difference is that a further two tail bits are appended to the input and interleaved sequences. Since the BCJR algorithm is used, an unterminated sequence (as in code $\mathrm{F}$ ) is equivalent to a terminated sequence where no tail information is available. Thus, the information advantage of code $\mathrm{G}$ over code $\mathrm{F}$ is the (complete) tail information for the input sequence and the tail parity information for the interleaved sequence. However, this extra information comes at a cost of code rate reduction by a factor of $\frac{66}{64}$. It would seem from the simulation results that the information advantage is offset by the code rate reduction for this particular interleaver. It can be seen from the uniform interleaver simulations, however, that on the average, the JPL termination scheme does provide a performance improvement. This advantage seems to depend on the particular interleaver used.

- In desigining an interleaver which self-terminates, we restrict the search space, effectively reducing the algorithm's ability at increasing the interleaver spread. For this reason, while code $\mathrm{H}$ has the advantage of being correctly-terminating (with complete tail information for both decoders), it not only suffers the penalty of code rate reduction (as for code $G$ ), but also a small reduction in spread.

In conclusion, while termination is beneficial for the average interleaver, its effect on optimised interleavers is negligible and not always beneficial. Considering that designing an interleaver without termination is simpler and that there is also a minor gain in code rate, the design of non-terminating interleavers should be preferred for all block sizes.

\section{Conclusions}

We have proposed a new interleaver design technique, and shown that it performs better than other regular and partly-randomised designs. For the case of small interleavers, it is shown that while correct termination improves the performance for an average interleaver, 
its effect on Turbo codes with optimised interleavers is negligible for codes with $\nu=2$. Considering that designing an interleaver without termination is simpler and that there is also a minor gain in code rate, the design of non-terminating interleavers should be preferred for all block sizes.

Using our simulated annealing design technique it is easier to include restrictions which make the interleaver correctly-terminating or odd-even. While the $S$-random algorithm serves well for specifying interleaver spread, we believe that our algorithm is better suited for more sophisticated design criteria.

Utilising some performance enhancement techniques, the complexity of the energy function grows only as $O(\tau)$, making it suitable for use with large block sizes. However, increasing the block size also requires the simulated annealing algorithm to perform more perturbations at every temperature, so that the overall complexity of the design technique actually grows larger than $O(\tau)$.

\section{Future WORK}

\section{A. Improving the Simulated Annealing Design}

While we have shown that simulated annealing can be used to design optimised interleavers, the energy functions used are based on the 'high spreading factor' criterion used by the JPL team in their $S$-random interleaver. Thus, the performance of codes with our interleavers are not expected to be significantly better than those using $S$-random interleavers.

More sophisticated energy functions will need to be considered if any further improvement is sought. Possible directions of research may include:

- Energy functions that take into consideration the fact that not all weight-two input sequences produce a finite-length parity sequence. Those sequences resulting in a parity of infinite weight should be given less importance since they will not adversely affect the Turbo code's weight spectrum.

- Consideration of sequences of weight greater than two which produce finite length parity sequences should also improve performance, particularly at the lower SNR values. This is, however, a problem of considerable complexity, and requires the energy function to be 
matched to the particular component codes used.

\section{B. Interleaver Design for Punctured Codes}

Our analysis has taken into consideration only unpunctured rate- $\frac{1}{3}$ symmetric Turbo codes. The problem of interleaver design for punctured codes also necessitates an appropriate interleaver optimisation. Some work has already been done on punctured codes, particularly indicating the usefulness of an odd-even interleaver structure. Modification of the simulated annealing algorithm to create odd-even interleavers is trivial, and should provide a preliminary design strategy. The effect of trellis termination on punctured codes needs to be investigated, particularly for small block sizes.

\section{REFERENCES}

[1] John L. Ramsey, "Realization of optimum interleavers," IEEE Transactions on Information Theory, vol. 16, no. 3, pp. 338-345, May 1970.

[2] Claude Berrou, Alain Glavieux, and Punja Thitimajshima, "Near Shannon limit error-correcting coding and decoding: Turbo-codes," in Proceedings of the IEEE International Conference on Communications, Geneva, Switzerland, May 1993, pp. 1064-1070.

[3] Dariush Divsalar and Fabrizio Pollara, "Turbo codes for deep-space communications," TDA progress report 42-120, Jet Propulsion Laboratory, California Institute of Technology, Feb. 15th, 1995.

[4] Dariush Divsalar and Fabrizio Pollara, "Multiple turbo codes for deep-space communications," TDA progress report 42-121, Jet Propulsion Laboratory, California Institute of Technology, May 15th, 1995.

[5] Lance C. Perez, Jan Seghers, and Daniel J. Costello, Jr., "A distance spectrum interpretation of turbo codes," IEEE Transactions on Information Theory, vol. 42, no. 6, pp. 1698-1709, Nov. 1996.

[6] Claude Berrou and Alain Glavieux, "Near optimum error correcting coding and decoding: Turbo-codes," IEEE Transactions on Communications, vol. 44, no. 10, pp. 1261-1271, Oct. 1996.

[7] P. Jung and M. Naßhan, "Dependence of the error performance of turbo-codes on the interleaver structure in short frame transmission systems," Electronics Letters, vol. 30, no. 4, pp. 287-288, Feb. 17th, 1994.

[8] Kenneth S. Andrews, Chris Heegard, and Dexter Kozen, "A theory of interleavers," Technical report tr971634, Department of Computer Science, Cornell University, June 1997.

[9] Kenneth S. Andrews, Chris Heegard, and Dexter Kozen, "Interleaver design methods for turbo codes," in Proceedings of the IEEE International Symposium on Information Theory, Cambridge, MA, USA, Aug. 1621st, 1998, p. 420.

[10] Johan Hokfelt, Ove Edfors, and Torleiv Maseng, "Interleaver design for turbo codes based on the performance of iterative decoding," in Proceedings of the IEEE International Conference on Communications, Vancouver, BC, Canada, June 6-10th, 1999.

[11] Jinhong Yuan, Branka Vucetic, and Wen Feng, "Combined turbo codes and interleaver design," in Proceedings of the IEEE International Symposium on Information Theory, Cambridge, MA, USA, Aug. 16-21st, 1998.

[12] Jinhong Yuan, Branka Vucetic, and Wen Feng, "Combined turbo codes and interleaver design," IEEE Transactions on Communications, vol. 47, no. 4, pp. 484-487, Apr. 1999. 
[13] Peter J. M. van Laarhoven and E. H. L. Aarts, Simulated Annealing: Theory and Applications, Mathematics and its Applications. D. Reidel Publishing Company, Holland, 1987.

[14] Johann A. Briffa, Interleavers for Turbo Codes, M.Phil. thesis, University of Malta, Faculty of Engineering, 1999.

[15] Dariush Divsalar and Fabrizio Pollara, "On the design of turbo codes," TDA progress report 42-123, Jet Propulsion Laboratory, California Institute of Technology, Nov. 15th, 1995.

[16] Sorin Adrian Barbulescu and Steven Silvio Pietrobon, "Terminating the trellis of turbo-codes in the same state," Electronics Letters, vol. 31, no. 1, pp. 22-23, Jan. 5th, 1995.

[17] Sorin Adrian Barbulescu, Iterative Decoding of Turbo Codes and Other Concatenated Codes, Ph.D. thesis, University of South Australia, School of Electronic Engineering, Faculty of Engineering, Feb. 1996. 


\section{procedure simulated annealing}

begin

Initialise state $X=X_{0}$

Initialise temperature $T=T_{0}$

repeat

repeat

Choose $X^{\prime}$, a perturbation of $X$

Let $\Delta E=\operatorname{Energy}\left(X^{\prime}\right)-\operatorname{Energy}(X)$

if $\Delta E<0$ or random $[0,1)<e^{-\frac{\Delta E}{T}}$

Change state $X=X^{\prime}$

\section{end if}

until several state changes or too many iterations

Decrease temperature according to annealing schedule until final temperature reached or configuration is stable end

Fig. 1. Simulated Annealing - Basic algorithm

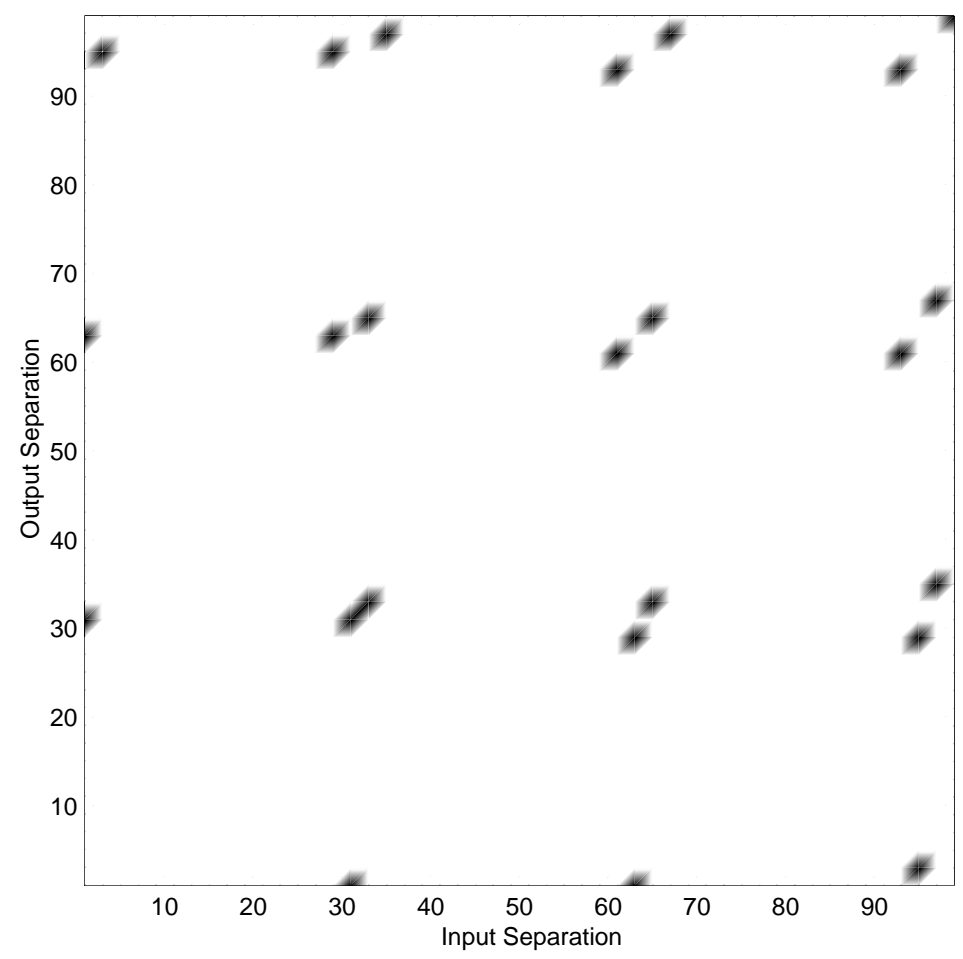

Fig. 2. IOSS for Square Interleaver 


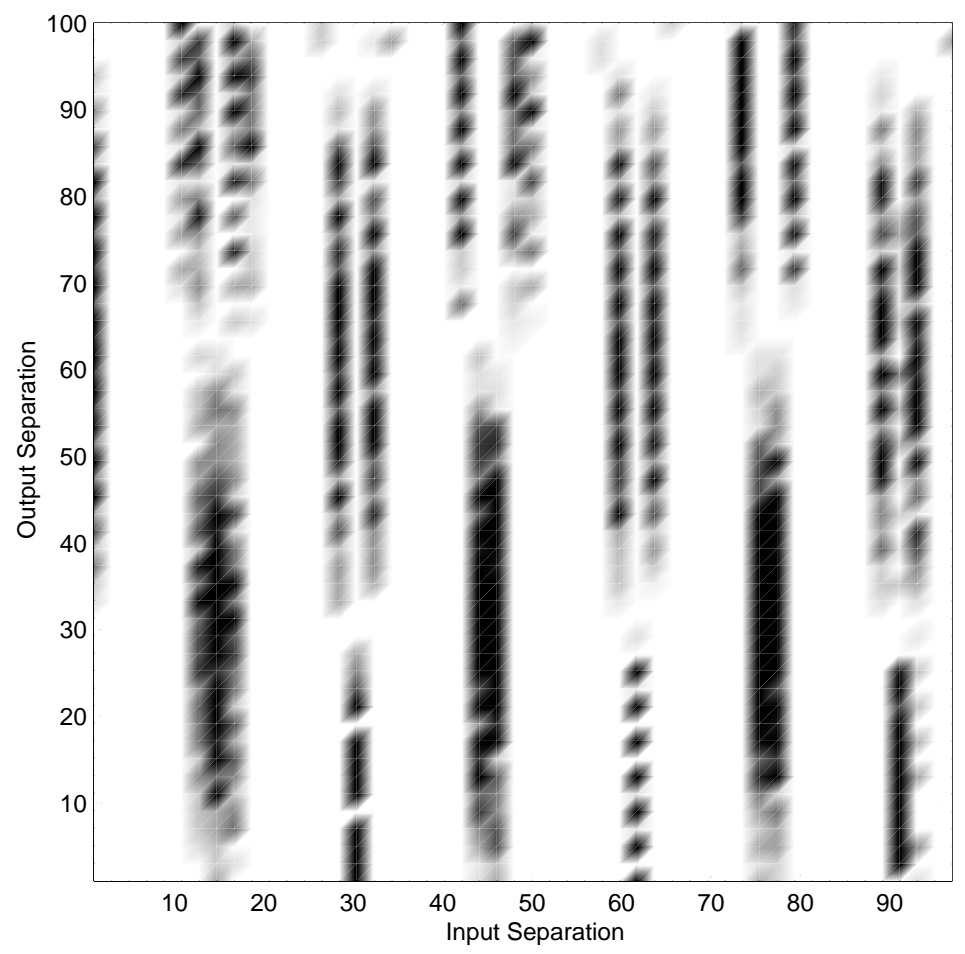

Fig. 3. IOSS for Berrou-Glavieux Interleaver

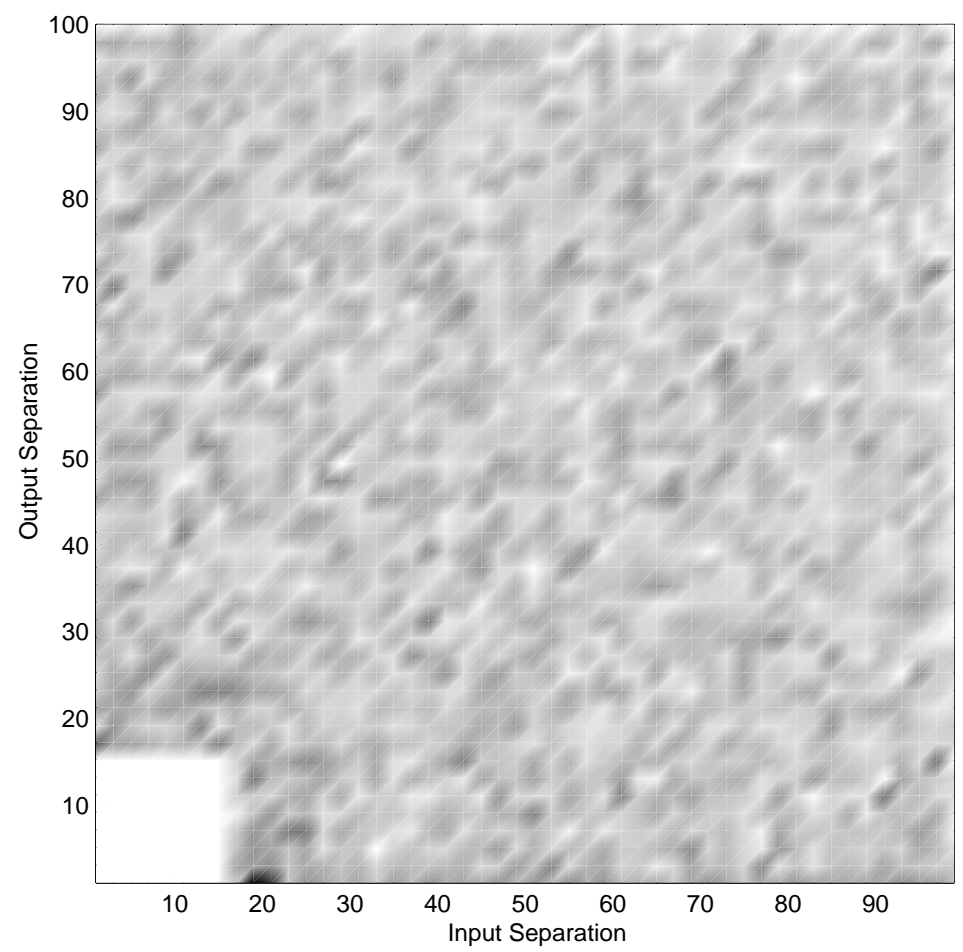

Fig. 4. IOSS for $S$-random Interleaver 


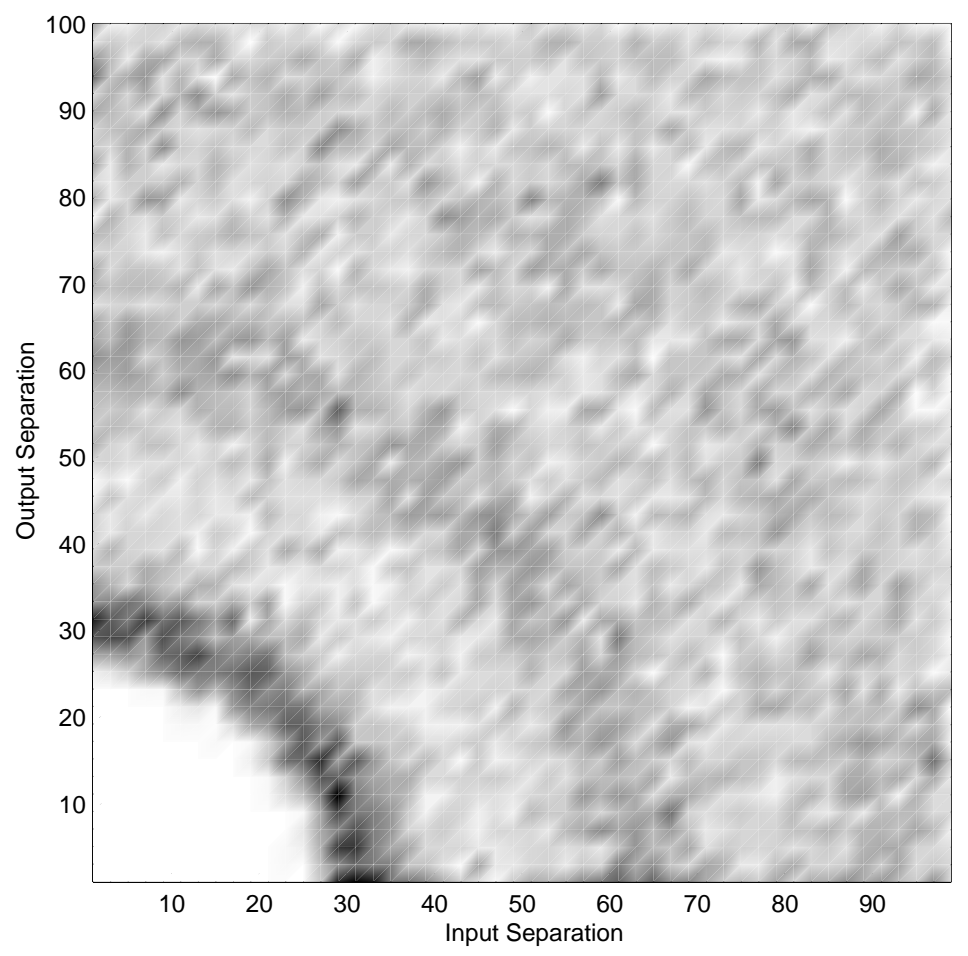

Fig. 5. IOSS for Simulated Annealing Interleaver

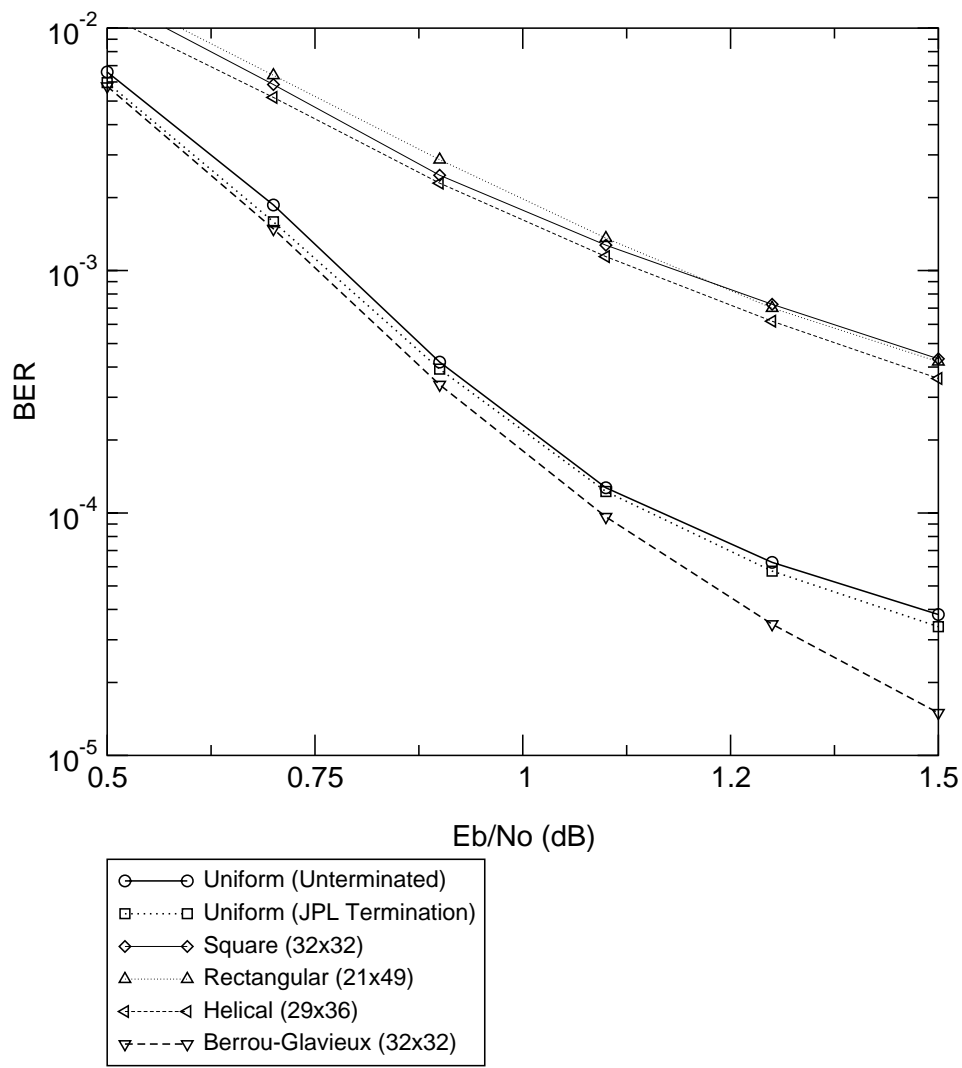

Fig. 6. Turbo code BER simulation (large block size) - Regular interleavers 


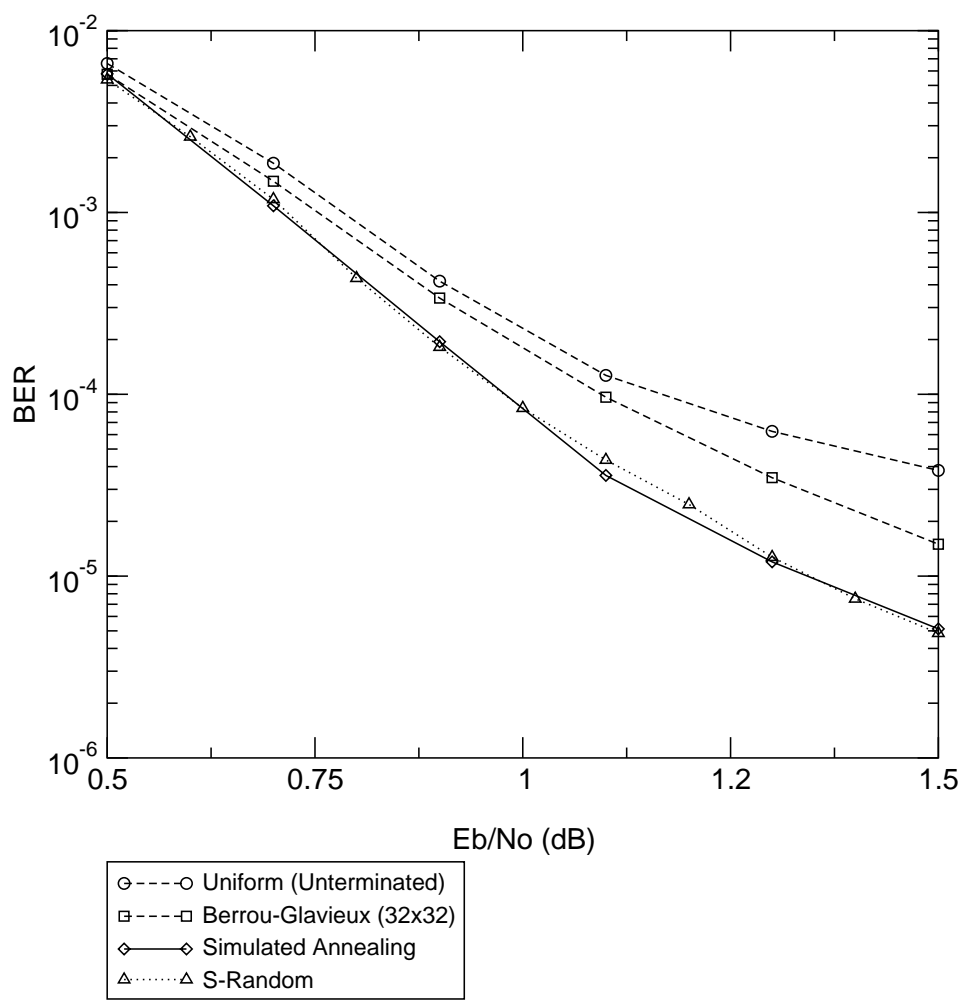

Fig. 7. Turbo code BER simulation - Optimised interleavers

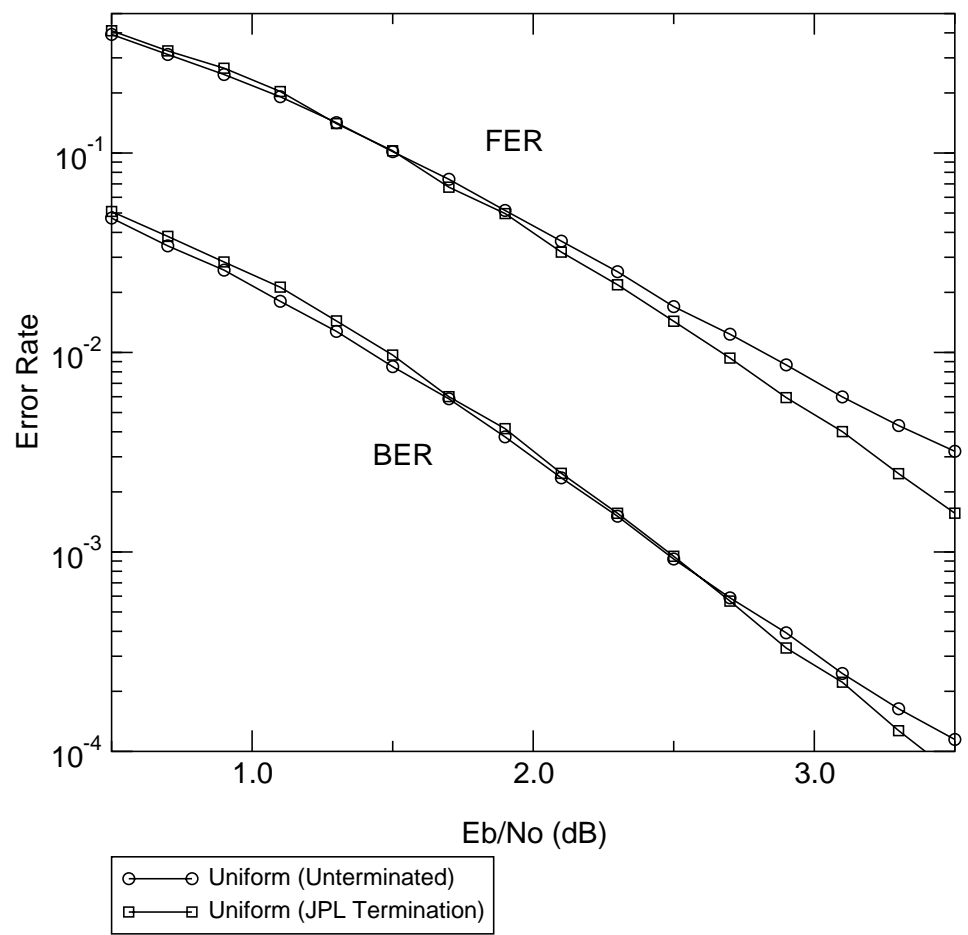

Fig. 8. Turbo code BER and FER simulation (small block size) - Uniform interleaver 


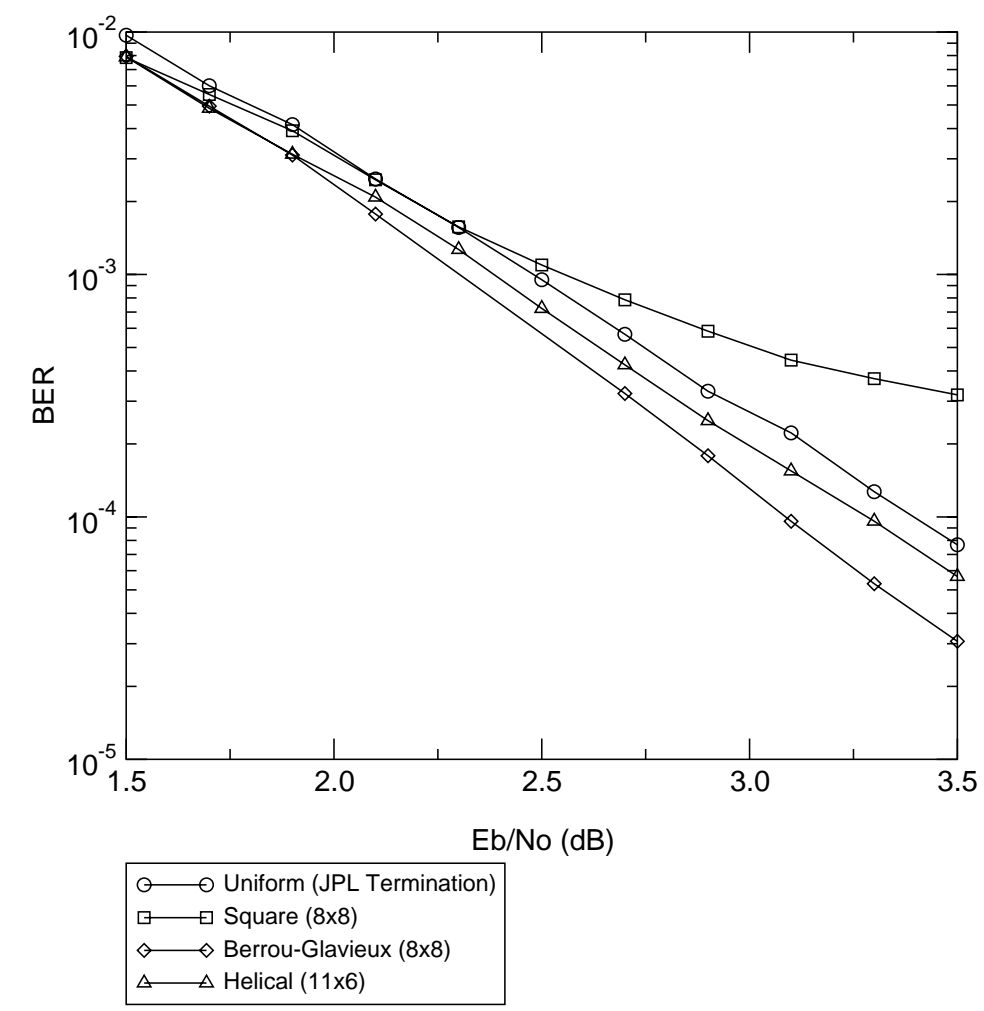

Fig. 9. Turbo code BER simulation (small block size) - Deterministic interleavers

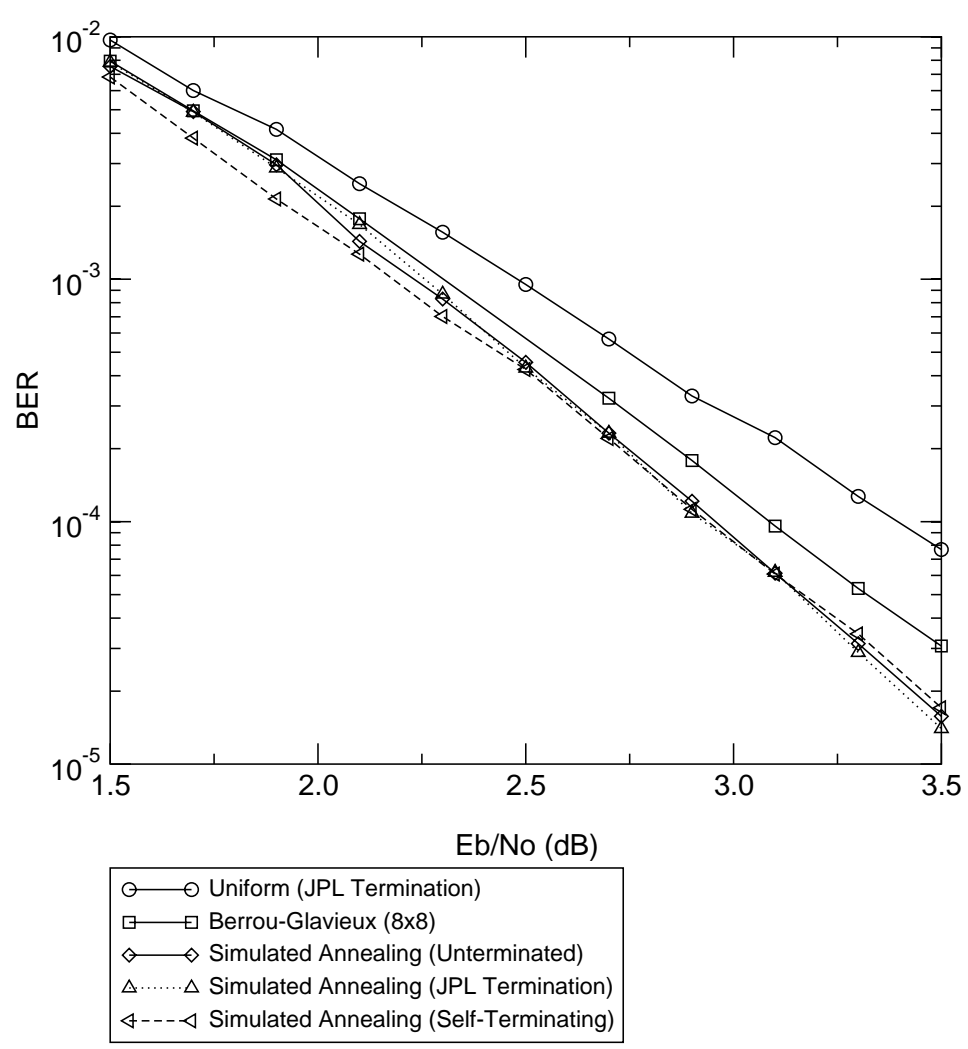

Fig. 10. Turbo code BER simulation (small block size) - Optimised interleavers 


\begin{tabular}{|c|c|c|c|c|c|}
\hline Label & Type & Parameters & Spread & Termination & Code Size \\
\hline A & Square & $32 \times 32$ & 31 & None & $(3072,1024)$ \\
B & Rectangular & $21 \times 49$ & 21 & None & $(3087,1029)$ \\
C & Helical & $29 \times 36$ & 1 & Simile & $(3138,1044)$ \\
D & Berrou-Glavieux & $32 \times 32$ & 14 & None & $(3072,1024)$ \\
\hline E & Uniform & (n/a) & (n/a) & None & $(3072,1024)$ \\
F & Uniform & (n/a) & (n/a) & JPL & $(3078,1024)$ \\
\hline G & Simulated Annealing & (see text) & 19 & None & $(3072,1024)$ \\
H & S-random Interleaver & (see text) & 18 & None & $(3072,1024)$ \\
\hline
\end{tabular}

TABLE I

INTERLEAVERS FOR A 1024-BIt Frame

\begin{tabular}{|c|c|c|c|c|}
\hline Label & Type & Parameters & Termination & Code Size \\
\hline A & Uniform & $(\mathrm{n} / \mathrm{a})$ & None & $(192,64)$ \\
B & Uniform & $(\mathrm{n} / \mathrm{a})$ & JPL & $(198,64)$ \\
\hline C & Square & $8 \times 8$ & None & $(192,64)$ \\
D & Berrou-Glavieux & $8 \times 8$ & None & $(192,64)$ \\
E & Helical & $11 \times 6$ & Simile & $(204,66)$ \\
\hline F & Simulated Annealing & (see text) & None & $(192,64)$ \\
G & Simulated Annealing & (see text) & JPL & $(198,64)$ \\
H & Simulated Annealing & (see text) & Self & $(198,64)$ \\
\hline
\end{tabular}

TABLE II

INTERLEAVERS FOR A 64-BIT FRAME 